11

\title{
Импедансные характеристики двухэлементной антенной решетки с киральной подложкой
}

\author{
(с) А.Л. Бузов ${ }^{1}$, Д.С. Клюев ${ }^{2,9}$, Д.А. Копылов ${ }^{1}$, А.М. Нещерет ${ }^{1}$ \\ ${ }^{1}$ AO „Самарское инновационное предприятие радиосистем“, Самара, \\ Россия \\ ${ }^{2}$ Поволжский государственный университет телекоммуникаций \\ и информатики, Самара, Россия \\ IE-mail: klyuevd@yandex.ru
}

Поступило в Редакцию 11 июля 2018 г.

Проведен расчет импедансных характеристик двухэлементной антенной решетки с подложкой из кирального метаматериала. Получена система сингулярных интегральных уравнений с особенностями типа Коши относительно неизвестных продольных распределений поверхностной плотности тока. Представлены зависимости входных импедансов от нормированной длины плеча для различных типов подложек, а также различных значений параметра киральности подложки.

DOI: 10.21883/PJTF.2018.23.47007.17459

Перспективным направлением в области разработки антенных систем нового поколения, обладающих улучшенными электрическими и массогабаритными характеристиками, является применение в их конструкции метаматериалов [1]. В частности, применение метаматериалов позволяет улучшить характеристики направленности антенн, уменьшить их, а также уменьшить взаимное влияние между излучателями в антенных решетках. Последнее достаточно важно при использовании подобных антенн с метаматериалами в системах MIMO (multiple input multiple output), позволяющих увеличить пропускную способность [2]. Кроме того, уменьшение взаимного влияния может также оказаться существенным при обеспечении электромагнитной совместимости группы радиосредств, которые работают на близких частотах и расположены на довольно малом объекте. 


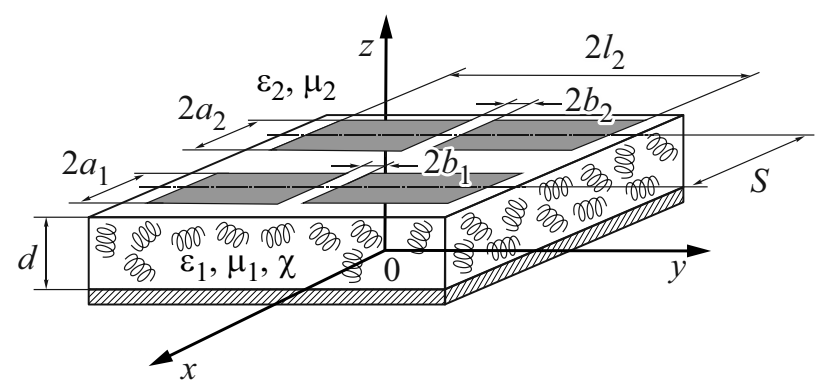

Рис. 1. Геометрия двухэлементной антенной решетки с киральной подложкой.

Вместе с тем в настоящее время существует актуальная проблема, связанная с электродинамическим анализом и синтезом подобных антенн, содержащих структуры из метаматериалов, поскольку отсутствуют их корректные физические и математические модели. В связи с этим, задача настоящей работы заключается в разработке адекватных математических моделей двухэлементных антенных решеток с киральными подложками, обеспечивающих корректный расчет их электрических характеристик.

Следует отметить, что разработанная модифицированная математическая модель выполнена на основе базовой модели микрополосковой структуры с одиночным излучателем, расположенным на киральной подложке [3]. В свою очередь такая базовая модель микрополосковой структуры была разработана на основе использования подхода сингулярного интегрального представления поля [4].

Рассмотрим антенную решетку (рис. 1), представляющую собой киральную подложку с односторонней металлизацией, толщина которой равна $d$. На подложке на расстоянии $S$ расположены два прямоугольных плоских бесконечно тонких излучателя длиной $2 l_{1}$ и $2 l_{2}$, ширина которых составляет $2 a_{1}$ и $2 a_{2}$ и много меньше как их длины, так и длины волны $\left(a_{1}, a_{2} \ll \lambda\right)$. Над излучателями расположено диэлектрическое полупространство. При этом макроскопические параметры киральной подложки равны $\varepsilon_{1}, \mu_{1}, \chi$, а диэлектрического полупространства $\varepsilon_{2}, \mu_{2}$, где $\varepsilon_{1}, \varepsilon_{2}, \mu_{1}, \mu_{2}$ - относительные диэлектрические и магнитные проницаемости подложки и полупространства соответственно, а $\chi-$ параметр киральности.

Письма в ЖТФ, 2018, том 44, вып. 23 
На излучателях располагаются зазоры толщиной $2 b_{1}$ и $2 b_{2}$, в которые подключены гармонические источники ЭДС, обеспечивающие такое распределение токов на излучателях, что формируемое ими электромагнитное поле удовлетворяет уравнениям Максвелла, условию излучения Зоммерфельда, а также граничным условиям

$$
\begin{gathered}
\eta_{y}^{1}\left(x,-l_{1}\right)=\eta_{y}^{1}\left(x,+l_{1}\right)=0, \\
\eta_{y}^{2}\left(x+S,-l_{2}\right)=\eta_{y}^{2}\left(x+S,+l_{2}\right)=0, \\
\mathbf{E}_{\tau}^{1}(x, y)=0, \quad x \in\left[-a_{1}, a_{1}\right], \quad y \in\left[-l_{1},-b_{1}\right] \cup\left[b_{1}, l_{1}\right], \\
\mathbf{E}_{\tau}^{2}(x+S, y)=0, \quad x+S \in\left[-a_{2}, a_{2}\right], \quad y \in\left[-l_{2},-b_{2}\right] \cup\left[b_{2}, l_{2}\right], \\
\mathbf{E}_{\tau}^{1}(x, y)=-\mathbf{E}_{\tau}^{e x t_{-}}, \quad x \in\left[-a_{1}, a_{1}\right], \quad y \in\left[-b_{1}, b_{1}\right], \\
\mathbf{E}_{\tau}^{2}(x+S, y)=-\mathbf{E}_{\tau}^{e x t_{-} 2}, \quad x+S \in\left[-a_{2}, a_{2}\right], \quad y \in\left[-b_{2}, b_{2}\right] .
\end{gathered}
$$

При этом учитывается также то, что функции распределения поверхностных плотностей токов являются непрерывными по всей длине излучателей, а тангенциальные составляющие сторонних электрических полей имеют по одной компоненте $\mathbf{E}_{\tau}^{\text {ext }}{ }^{1}=\left(0, E_{y}^{e x t}-1,0\right)$ и $\mathbf{E}_{\tau}^{\text {ext_2 }}=\left(0, E_{y}^{\text {ext-2 }}, 0\right)$.

Как и в случае базовой модели, фурье-образы $\mathbf{T}_{\tau}=\left\{T_{x}, T_{y}\right\}$ тангенциальных составляющих напряженностей электрических полей $\mathbf{E}_{\tau}$ и фурье-образы $\mathbf{F}=\left\{F_{x}, F_{y}\right\}$ поверхностных плотностей токов $\boldsymbol{\eta}$ для соответствующих излучателей связаны через матрицу поверхностных импедансов $[Z]$

$$
\left[\begin{array}{l}
T_{y} \\
T_{x}
\end{array}\right]=\left[\begin{array}{ll}
Z_{11} & Z_{12} \\
Z_{21} & Z_{22}
\end{array}\right]\left[\begin{array}{l}
F_{y} \\
F_{x}
\end{array}\right] .
$$

Однако необходимо иметь в виду, что в силу разной ширины излучателей значения элементов матриц поверхностных импедансов могут иметь серьезные различия. Поэтому для каждого излучателя необходимо определить соответствующую матрицу. Методика определения матрицы поверхностных импедансов границы раздела диэлектрик-киральная среда приведена в работах $[3,4]$.

В связи с отмеченным выше разложим векторы напряженностей электрического $\mathbf{E}$ и магнитного Н полей и поверхностных плотностей токов $\boldsymbol{\eta}$ на излучателях по координатам $x$ и $y$ в интегралы Фурье, в результате чего с учетом матрицы поверхностных импедансов (2), а также

Письма в ЖТФ, 2018, том 44, вып. 23 
того, что ввиду малой ширины излучателей поперечной составляющей плотности тока $\eta_{x}$ можно пренебречь, получим следующую систему интегральных представлений поля на поверхности излучателей:

$$
\begin{aligned}
& \left\{\begin{array}{l}
E_{y}^{1}(0, y)=\int_{-l_{1}}^{l_{1}} \int_{-a_{1}}^{a_{1}} \eta_{y}^{1}\left(x^{\prime}, y^{\prime}\right) Z_{11}^{\Sigma_{-}}\left(x^{\prime}, y^{\prime} ; 0, y\right) d x^{\prime} d y^{\prime} \\
+\int_{-l_{2}}^{l_{2}} \int_{-a_{2}}^{S+a_{2}} \eta_{y}^{2}\left(x^{\prime \prime}, y^{\prime \prime}\right) Z_{11}^{\Sigma_{-} 2}\left(x^{\prime \prime}, y^{\prime \prime} ; 0, y\right) d x^{\prime \prime} d y^{\prime \prime}, \\
E_{y}^{2}(S, y)=\int_{-l_{1}}^{l_{1}} \int_{-a_{1}}^{a_{1}} \eta_{y}^{1}\left(x^{\prime}, y^{\prime}\right) Z_{11}^{\Sigma_{-} 1}\left(x^{\prime}, y^{\prime} ; S, y\right) d x^{\prime} d y^{\prime} \\
+\int_{-l_{2}}^{l_{2}} \int_{-a_{2}}^{S+a_{2}} \eta_{y}^{2}\left(x^{\prime \prime}, y^{\prime \prime}\right) Z_{11}^{\Sigma_{-} 2}\left(x^{\prime \prime}, y^{\prime \prime} ; S, y\right) d x^{\prime \prime} d y^{\prime \prime},
\end{array}\right. \\
& Z_{11}^{\Sigma_{-1}}\left(x^{\prime}, y^{\prime} ; x, y\right)=\frac{1}{4 \pi^{2}} \int_{-\infty}^{\infty} \int_{-\infty}^{\infty} Z_{11}^{1}(\beta, h) e^{-i \beta\left(x-x^{\prime}\right)} e^{-i h\left(y-y^{\prime}\right)} d \beta d h, \\
& Z_{11}^{\Sigma_{-} 2}\left(x^{\prime \prime}, y^{\prime \prime} ; x, y\right)=\frac{1}{4 \pi^{2}} \int_{-\infty}^{\infty} \int_{-\infty}^{\infty} Z_{11}^{2}\left(\beta^{\prime}, h^{\prime}\right) e^{-i \beta\left(x-x^{\prime \prime}\right)} e^{-i h^{\prime}\left(y-y^{\prime \prime}\right)} d \beta^{\prime} d h^{\prime} .
\end{aligned}
$$

В этих выражениях предполагалось, что координата по оси абсцисс источника ЭДС, располагаемого на первом излучателе, совпадает с началом координат, т.е. равна нулю $(x=0)$, а координата по оси абсцисс источника ЭДС, располагаемого на втором излучателе, равна расстоянию между рассматриваемыми излучателями, т.е. равна $S$ $(x=S)$.

В связи с тем, что излучатели обладают малой шириной, поперечную вариацию продольного распределения поверхностной плотности тока $\eta_{y}(x)$ можно описать в виде квазистатического распределения $1 / \sqrt{1-(x / a)^{2}}$, в результате чего функции распределения плотностей токов $\eta_{y}^{1}\left(x^{\prime}, y^{\prime}\right)$ и $\eta_{y}^{2}\left(x^{\prime \prime}, y^{\prime \prime}\right)$ будут описываться следующим образом:

$$
\eta_{y}^{1}\left(x^{\prime}, y^{\prime}\right)=\frac{f^{1}\left(y^{\prime}\right)}{\sqrt{1-\left(x^{\prime} / a\right)^{2}}}, \quad \eta_{y}^{2}\left(x^{\prime \prime}, y^{\prime \prime}\right)=\frac{f^{2}\left(y^{\prime \prime}\right)}{\sqrt{1-\left(x^{\prime \prime}-S / a_{2}\right)^{2}}},
$$

где $f^{1}\left(y^{\prime}\right)$ и $f^{2}\left(y^{\prime \prime}\right)$ - неизвестные функции, характеризующие продольные распределения поверхностных плотностей токов в первом и втором излучателях. 

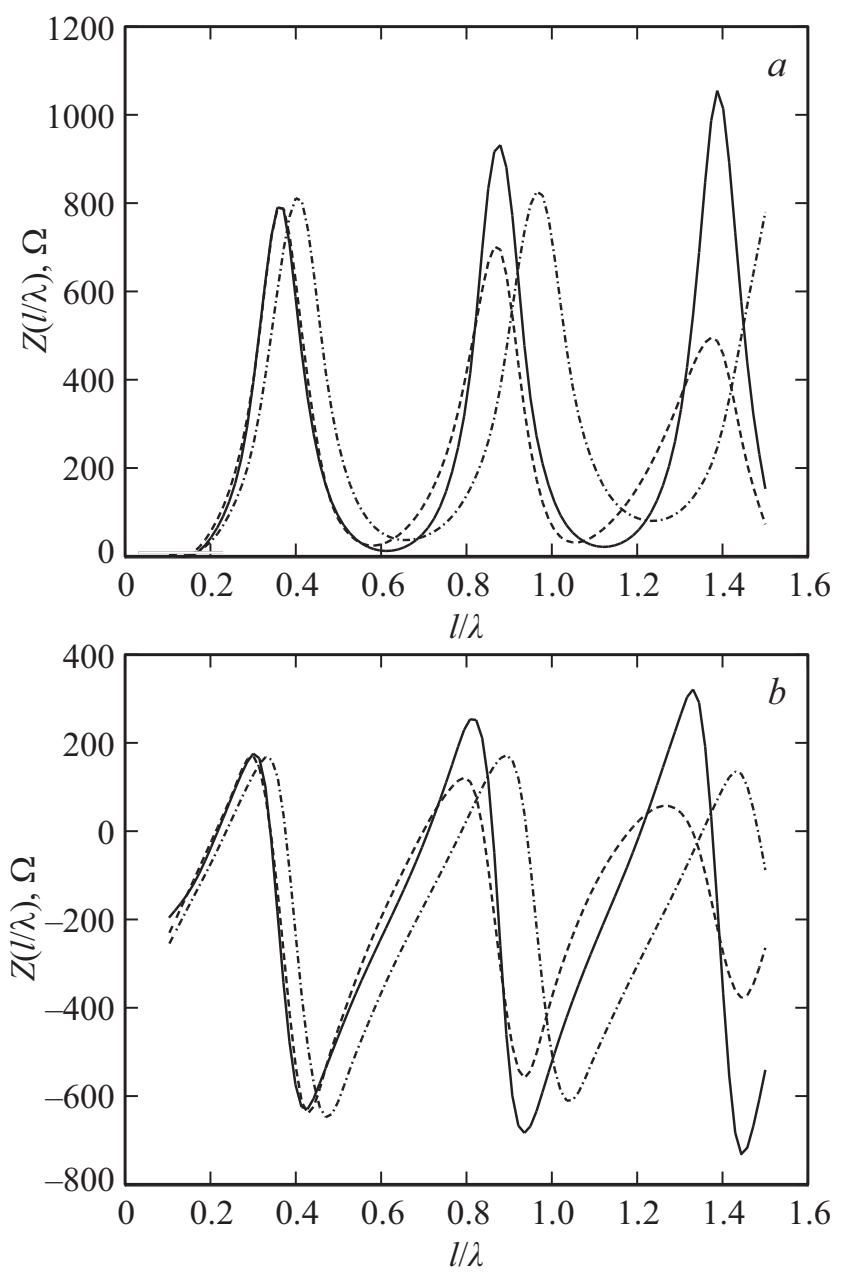

Рис. 2. Зависимость от длины плеча излучателя, нормированной на длину волны, действительной $(a, c)$ и мнимой $(b, d)$ частей входного импеданса излучателя антенной решетки, расположенного на диэлектрической $(\chi=0)$ (сплошная линия) и киральной подложках на основе право- (штриховая линия) и левосторонних (штрихпунктирная линия) элементов при параметре киральности $\chi=0.2(a, b)$ и $0.5(c, d)$.

Письма в ЖТФ, 2018, том 44, вып. 23 

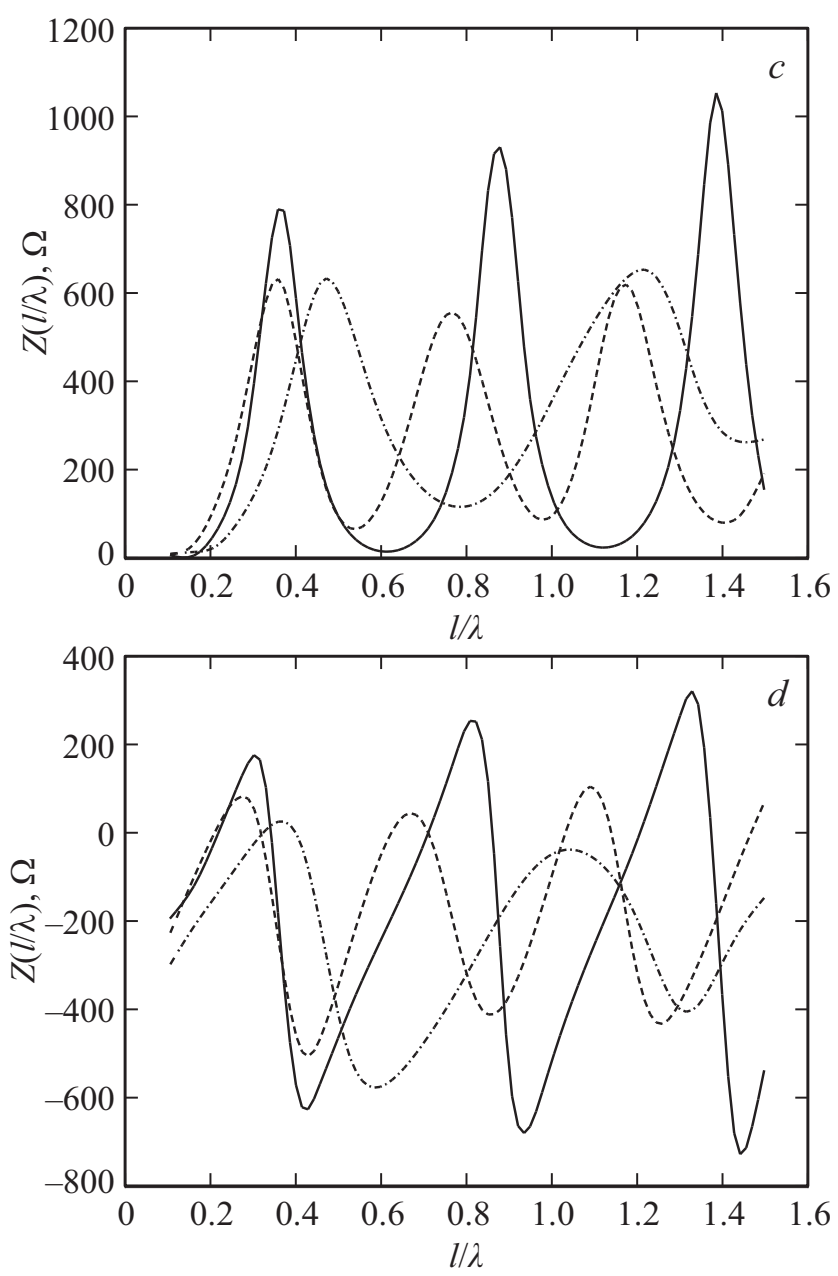

Рис. 2 (продолжение).

После подстановки функций плотностей токов (4) в (3), а также применения к получившимся выражениям операции интегрирования по частям по $y^{\prime}$ и $y^{\prime \prime}$ и учета граничных условий (1) перейдем к новым функциям $f^{\prime 1}\left(y^{\prime}\right)=d f^{1}\left(y^{\prime}\right) / d y^{\prime}$ и $f^{\prime 2}\left(y^{\prime \prime}\right)=d f^{2}\left(y^{\prime \prime}\right) / d y^{\prime \prime}$. Устраняя

Письма в ЖТФ, 2018, том 44, вып. 23 
расходимость в несобственных интегралах (3) по $h$ путем вычитания асимптотических сомножителей $Z_{11}^{\infty-1}(h)$ и $Z_{11}^{\infty-2}\left(h^{\prime}\right)$ и выполняя ряд аналитических преобразований, получим систему сингулярных интегральных представлений (СИП) электрического поля. Затем, подставляя в получившуюся систему СИП граничные условия (1), перейдем к системам сингулярных интегральных уравнений (СИУ) с особенностями Коши относительно неизвестных функций распределений плотностей токов $f^{\prime 1}\left(y^{\prime}\right)$ и $f^{\prime 2}\left(y^{\prime \prime}\right)$ по излучателям

$$
\begin{aligned}
& -E_{y}^{e x t_{-}}(0, y)=-\frac{a_{1}}{4 \pi} \int_{-l_{1}}^{l_{1}} f^{\prime 1}\left(y^{\prime}\right) \int_{-\infty}^{\infty} \int_{-\infty}^{\infty} \frac{1}{i h} J_{0}\left(\beta a_{1}\right) \\
& \times\left[Z_{11}^{1}(\beta, h)-C|h|\right] e^{-i h\left(y-y^{\prime}\right)} d \beta d h d y^{\prime}-\frac{C}{\pi} \int_{-l_{1}}^{l_{1}} \frac{f^{\prime 1}\left(y^{\prime}\right)}{y^{\prime}-y} d y^{\prime} \\
& -\frac{a_{2}}{4 \pi} \int_{-l_{2}}^{l_{2}} f^{\prime 2}\left(y^{\prime \prime}\right) \int_{-\infty}^{\infty} \int_{-\infty}^{\infty} \frac{1}{i h^{\prime}} J_{0}\left(\beta^{\prime} a_{2}\right) e^{-i \beta^{\prime} S} \\
& \times\left[Z_{11}^{2}\left(\beta^{\prime}, h^{\prime}\right)-C\left|h^{\prime}\right|\right] e^{-i h^{\prime}\left(y-y^{\prime \prime}\right)} d \beta^{\prime} d h^{\prime} d y^{\prime \prime}, \\
& -E_{y}^{e x t-2}(S, y)=-\frac{a_{1}}{4 \pi} \int_{-l_{1}}^{l_{1}} f^{\prime 1}\left(y^{\prime}\right) \int_{-\infty}^{\infty} \int_{-\infty}^{\infty} \frac{1}{i h} J_{0}\left(\beta a_{1}\right) e^{-i \beta S} \\
& \times\left[Z_{11}^{1}(\beta, h)-C|h|\right] e^{-i h\left(y-y^{\prime}\right)} d \beta d h d y^{\prime} \\
& a_{-\infty}^{\infty} \int_{-\infty}^{\infty} \frac{1}{i h^{\prime}} J_{0}\left(\beta^{\prime} a_{2}\right) \\
& \times\left[Z_{11}^{2}\left(\beta^{\prime}, h^{\prime}\right)-C\left|h^{\prime}\right|\right] e^{-i h^{\prime}\left(y-y^{\prime}\right)} d \beta^{\prime} d h^{\prime} d y^{\prime \prime}-\frac{C}{\pi} \int_{-l_{2}}^{l_{2}} \frac{f^{\prime 2}\left(y^{\prime \prime}\right)}{y^{\prime \prime}-y} d y^{\prime \prime} .
\end{aligned}
$$

Решение приведенной выше системы СИУ было проведено методом моментов в разложении функций распределений плотностей токов по

Письма в ЖТФ, 2018, том 44, вып. 23 
полиномам Чебышева первого рода. Более подробное решение приведено в [5]. В результате решения данной системы СИУ определяются функции, характеризующие продольные распределения поверхностных плотностей тока по излучателям. В свою очередь входной импеданс определяется в соответствии со следующим выражением:

$$
Z=\frac{V}{I_{y}\left(y^{\prime}=0\right)},
$$

где

$$
I_{y}\left(y^{\prime}\right)=\int_{-a}^{a} \eta_{y}\left(x^{\prime}, y^{\prime}\right) d x=\int_{-a}^{a} \frac{f\left(y^{\prime}\right)}{\sqrt{1-\left(x^{\prime} / a\right)^{2}}} d x^{\prime}=a \pi f\left(y^{\prime}\right),
$$

a $V=1-$ эффективная ЭДС на зажимах излучателя.

Далее представлены зависимости от длины плеча излучателя, нормированной на длину волны, действительной (рис. 2,a) и мнимой (рис. $2, b$ ) частей входного импеданса излучателя двухэлементной антенной решетки с диэлектрической подложкой $(\chi=0)$ и подложкой из кирального метаматериала на основе лево- и правосторонних элементов со следующими параметрами: $\varepsilon_{1}=\varepsilon_{2}=1, \mu_{1}=\mu_{2}=1, d / \lambda=0.1$, $S / \lambda=0.25, \chi=0.2$. При этом геометрические параметры излучателей равны: $a_{1} / \lambda=a_{2} / \lambda=0.025, b_{1} / \lambda=b_{2} / \lambda=0.01$, где $\lambda-$ длина волны. Кроме того, приведены зависимости действительной (рис. 2,c) и мнимой (рис. $2, d$ ) частей входного импеданса для параметров киральности $\chi=0$ и 0.5 при прочих равных остальных параметрах. В связи с тем, что излучатели абсолютно идентичны и запитаны синфазно, зависимости входного импеданса приводятся только для одного излучателя.

В результате анализа представленных зависимостей входного импеданса двухэлементной антенной решетки с киральной подложкой от длины плеча излучателя, нормированной на длину волны, установлено, что в случае киральной подложки на основе левосторонних элементов наблюдается уменьшение добротности. Кроме того, имеет место эффект укорочения. 


\section{Список литературы}

[1] Бузов А.Л., Клюев Д.С., Нещерет А.М., Неганов В.А. // Физика волновых процессов и радиотехнические системы. 2017. Т. 20. № 3. С. 15-20.

[2] Беспалов А.Н., Букашкин С.А., Назин В.Ю., Нещерет А.М., Телегин С.С. // Радиотехника. 2018. № 4. С. 87-91.

[3] Бузова М.А., Клюев Д.С., Минкин М.А., Нещерет А.М., Соколова Ю.В. // Письма в ЖТФ. 2018. Т. 44. В. 11. С. 80-86.

[4] Дементьев А.Н., Клюев Д.С., Неганов В.А., Соколова Ю.В. Сингулярные и гиперсингулярные интегральные уравнения в теории зеркальных и полосковых антенн. М.: Радиотехника, 2015. 216 с.

[5] Аронов В.Ю., Клюев Д.С., Копылов Д.А., Нещерет А.М. // Радиотехника. 2018. № 4. C. 64-69. 